\title{
De-escalation of empiric antibiotic therapy in sepsis - an indian observational study
}

\author{
S Jakkinaboina*, K Swarna Deepak \\ From ESICM LIVES 2015 \\ Berlin, Germany. 3-7 October 2015
}

\section{Introduction}

The guidelines for an appropriate use of antibiotic therapy recommend de-escalation as soon as the culture sensitivity results are available.There was a great need to study the frequency of de-escalation, no change and escalation of antibiotics and its impact in septic patients in an Indian ICU setting in view of rising burden of drug resistance and health care cost.

\section{Objectives}

To determine the rate of de-escalation of the empiric antimicrobial treatment in ICU patients admitted with sepsis. The characteristics of antimicrobial treatment associated with de-escalation \& its impact on ICU length of stay, hospital length of stay (LOS) and 30 day mortality were determined.

\section{Methods}

We carried out a prospective observational study enrolling patients admitted to the ICU with sepsis from 01/02/2014 to $01 / 03 / 2015$. Both the medical and surgical units of the
ICU were included. "No change" was defined as when empiric therapy was maintained without modification, "Escalation of therapy" was defined when there's a switch to or addition of an antibiotic with a broader spectrum, and "De-escalation" when there's a switch to or interruption of a drug class resulting in a less broad spectrum of coverage. If antimicrobial change consisted of escalation and de-escalation (i.e. switch to or addition of an antibiotic with a broader spectrum but also withdrawal of another antibiotic), the patient was assigned to "escalation group" for statistical analysis.

\section{Results}

The total number of patients enrolled were 315 out of which 17 patients were excluded due to death before culture results were available. The characteristics observed among the therapeutic strategy groups were seen in tables $1,2$.

The most common antibiotic after de-escalation\& antibiotic in No Change group was Betalactam + beta lactamase inhibitor.

Table 1. Patient characteristics with therapeutic strategy

\begin{tabular}{|c|c|c|c|c|}
\hline Variable & No change $n$ & De-escalation $\mathbf{n}$ & Escalation $\mathbf{n}$ & $P$ value \\
\hline Number of patients & 148 & 74 & 76 & \\
\hline Age in years & 57.62 & 57.81 & 58.18 & 0.96 \\
\hline HOSPITAL length of stay in days & 11.41 & 10.68 & 19.21 & 0.0001 \\
\hline APACHE II score at admission & 15.3 & 15.84 & 20.11 & 0.0001 \\
\hline SOFA score at admission & 6.07 & 6.65 & 8.08 & 0.004 \\
\hline Number of patients expired at 30 days after admission & 20 & 10 & 26 & 0.0001 \\
\hline number of medical patients & 144 & 64 & 66 & 0.003 \\
\hline Number of patients requiring vasopressors & 20 & 16 & 34 & 0.0001 \\
\hline ICU length of stay in days & 5.85 & 6.24 & 12.42 & 0.0001 \\
\hline
\end{tabular}


Table 2. Patient characteristics with therapeutic strategy

\begin{tabular}{|c|c|c|c|c|}
\hline Variable & No change $n$ & De-escalation $\mathrm{n}$ & Escalation $n$ & $\begin{array}{l}P \\
\text { value }\end{array}$ \\
\hline Number of initial empirical antibiotics 1 & 58 & 26 & 32 & 0.007 \\
\hline Number of initial empirical antibiotics 2 & 72 & 26 & 36 & \\
\hline Number of initial empirical antibiotics 3 & 18 & 22 & 8 & \\
\hline Most Common initial empirical antibiotic & $\begin{array}{l}\text { beta lactam +beta lactamase } \\
\text { inhibitor }\end{array}$ & Carbapenem & $\begin{array}{l}\text { beta lactam +beta lactamase } \\
\text { inhibitor }\end{array}$ & 0.0001 \\
\hline $\begin{array}{l}\text { Most Common Escalated /De-escalated } \\
\text { Antibiotic }\end{array}$ & $\begin{array}{l}\text { beta lactam +beta lactamase } \\
\text { inhibitor }\end{array}$ & $\begin{array}{l}\text { beta lactam +beta lactamase } \\
\text { inhibitor }\end{array}$ & $\begin{array}{l}\text { Carbapenem followed by } \\
\text { colistin }\end{array}$ & 0.0001 \\
\hline Multi drug resistant organisms & 6 & 8 & 34 & 0.0001 \\
\hline ESBL organisms & 4 & 4 & 10 & 0.0001 \\
\hline Most Common Source of infection & Lung & Lung & Lung & \\
\hline H1N1 POSITIVE patients & 29 & 10 & 6 & 0.062 \\
\hline
\end{tabular}

\begin{tabular}{|c|c|c|}
\hline Variable & Mean $n$ & Standard deviation (W) \\
\hline Age in years & 57.81 & 15.34 \\
\hline ICU length of stay in days & 7.62 & 7.98 \\
\hline $\begin{array}{l}\text { HOSPITAL length of stay in } \\
\text { days }\end{array}$ & 13.21 & 11,43 \\
\hline APACHE II score at admission & 16.65 & 7.83 \\
\hline SOFA Score at admission & 6.72 & 4.35 \\
\hline Number of Male patients & 172 & $(57.72)$ \\
\hline Mortality at 30 days & 56 & $(18.79)$ \\
\hline Number of medical patients & 274 & (91.95) \\
\hline Number of Surgical Patients. & 24 & $(8.05]$ \\
\hline $\begin{array}{l}\text { Number of patients with } \\
\text { Neutropenia }\end{array}$ & 12 & 4.03 \\
\hline $\begin{array}{l}\text { Number of Patients who } \\
\text { required Vasopressors }\end{array}$ & 70 & $(23,49)$ \\
\hline Source as LUNG & 186 & $(62.42)$ \\
\hline Source as UROGENITAL TRACT & 42 & $(14.09)$ \\
\hline $\begin{array}{l}\text { Number of patients with MDR } \\
\text { organisms }\end{array}$ & 48 & $(16.1)$ \\
\hline $\begin{array}{l}\text { Number of H1N1 Positive } \\
\text { patients }\end{array}$ & 45 & (15.1) \\
\hline $\begin{array}{l}\text { Patients started with initial } \\
\text { number of empirical } \\
\text { antibiotics - } 1 \\
1 \\
2 \\
3\end{array}$ & $\begin{array}{l}116 \\
116 \\
194 \\
48\end{array}$ & $\begin{array}{l}(38.93) \\
(44.97) \\
(16.11)\end{array}$ \\
\hline $\begin{array}{l}\text { Initial Empirical Antibiatic } \\
\text { Betalactam + Betalactam ase } \\
\text { Inhibitor } \\
\text { Carbapenems }\end{array}$ & $\begin{array}{l}134 \\
78\end{array}$ & $\begin{array}{l}(44.97) \\
(26.17)\end{array}$ \\
\hline $\begin{array}{l}\text { THERAPEUTIC STRATEGY } \\
\text { APPLIED } \\
\text { No Change } \\
\text { De Escalation } \\
\text { Escalation }\end{array}$ & $\begin{array}{l}148 \\
74 \\
76 \\
\end{array}$ & $\begin{array}{l}(49.65) \\
(24.83) \\
(25.5) \\
\end{array}$ \\
\hline
\end{tabular}

Figure 1 The characteristics of the overall group of patients.

The statistically significant factors which increased the 30 day mortality are Escalation of antibiotics, Increased ICU LOS, Increased APACHE II and SOFA scores at admission, medical patients, vasopressor requirement, lung as the source of infection, Infection with MDR organisms.

The statistically significant factors which increased the ICU LOS are use of vasopressors, Comorbidities, 


\begin{tabular}{|c|c|c|c|}
\hline VARIABLE & $\begin{array}{l}\text { NON SU RVIVORS AT } \\
30 \text { DAYS } n\end{array}$ & $\begin{array}{l}\text { SURVIVORS AT } 30 \\
\text { DAYS } n\end{array}$ & PVALUE \\
\hline AGE in Years & 59.86 & 57.34 & 0.29 \\
\hline $\begin{array}{l}\text { ICU length of stay in } \\
\text { days }\end{array}$ & 9.75 & 7.13 & 0.027 \\
\hline $\begin{array}{l}\text { Hospital length of } \\
\text { stay in days }\end{array}$ & 15.29 & 12.74 & 0.13 \\
\hline $\begin{array}{l}\text { SOFA score at } \\
\text { admissian }\end{array}$ & 9.64 & 6.05 & 0.0001 \\
\hline $\begin{array}{l}\text { Number of Male } \\
\text { patients }\end{array}$ & 40 & 132 & 0.021 \\
\hline $\begin{array}{l}\text { Number of Medical } \\
\text { patients }\end{array}$ & 52 & 222 & 0.781 \\
\hline $\begin{array}{l}\text { Number of patients } \\
\text { with Neutropenia }\end{array}$ & 2 & 10 & 0.847 \\
\hline $\begin{array}{l}\text { Number of patients } \\
\text { who required } \\
\text { Vasopressors }\end{array}$ & 26 & 44 & 0.0001 \\
\hline $\begin{array}{l}\text { Number of patients } \\
\text { with Lung as source }\end{array}$ & 40 & 146 & \\
\hline $\begin{array}{l}\text { Number of patients } \\
\text { with MDR organisms }\end{array}$ & 20 & 30 & 0.0001 \\
\hline $\begin{array}{l}\text { Number of patients } \\
\text { with ESBL organisms }\end{array}$ & 0 & 10 & 0.0001 \\
\hline $\begin{array}{l}\text { Number of H1N1 } \\
\text { infection }\end{array}$ & 8 & 37 & 0.85 \\
\hline $\begin{array}{l}\text { Patients with initial } \\
\text { number of antibiotics } \\
1 \\
2 \\
3 \\
\end{array}$ & $\begin{array}{l}22 \\
22 \\
12 \\
\end{array}$ & $\begin{array}{l}94 \\
112 \\
36\end{array}$ & 0.425 \\
\hline $\begin{array}{l}\text { Therapeutic Strategy } \\
\text { Applied } \\
\text { No Change } \\
\text { De escalation } \\
\text { Escalation }\end{array}$ & $\begin{array}{l}20 \\
10 \\
26\end{array}$ & $\begin{array}{l}128 \\
64 \\
50\end{array}$ & 0.0001 \\
\hline
\end{tabular}

Figure 2 Comparison between survivors and non survivors at 30 days.

Table 3 Prevalence of organisms in patient groups. The frequency of organisms in culture positive cases.

\begin{tabular}{lllll}
\hline ORGANISM ISOLATED & NO CHANGE $\mathbf{n}$ & DE ESCALATION $\mathbf{n}$ & ESCALATION $\mathbf{n}$ & Total $\mathbf{n}$ (\%) \\
\hline Fungal infections & 4 & 2 & 8 & $14(9.39 \%)$ \\
\hline Non ESBL Gram Negative organisms & 12 & 10 & 8 & $30(20.13 \%)$ \\
\hline ESBL Gram negative organisms & 4 & 4 & 10 & $18(12.08 \%)$ \\
\hline MDR organisms & 6 & 8 & 34 & $48(32.21 \%)$ \\
\hline MRSA organisms & 0 & 6 & 0 & $6(4.02 \%)$ \\
\hline MSSA organisms & 0 & 2 & 2 & $4(2.68 \%)$ \\
\hline Vancomycin Resistant Enterococcus & 2 & 0 & 0 & $2(1.34 \%)$ \\
\hline Stenotrophomonas maltophila & 4 & 4 & 4 & $12(8.05 \%)$ \\
\hline
\end{tabular}


APACHE II at admission, SOFA at admission, MDR organisms, escalation of antibiotic.

\section{Conclusions}

Escalation of antibiotics resulted in statistically significant increase in the mortality and increased length of stay in the ICU and hospital. No significant differences were observed with respect to ICU length of stay, Hospital length of stay and 30 day mortality among No Change \& De Escalation groups.

Published: 1 October 2015

\section{Reference}

1. Garnacho-Montero J, Gutiérrez-Pizarraya A, Escoresca-Ortega A, Corcia-

Palomo Y, Fernández-Delgado E, Herrera-Melero I, et al: De-escalation of empirical therapy is associated with lower mortality in patients with severe sepsis and septic shock. Intensive Care Med 2014, 40:32-40.

\section{Submit your manuscript to a SpringerOpen ${ }^{\circ}$ journal and benefit from:}

- Convenient online submission

- Rigorous peer review

- Immediate publication on acceptance

- Open access: articles freely available online

- High visibility within the field

- Retaining the copyright to your article 